\title{
Performance of integrated pulmonary index for pulmonary embolism in dyspneic patients
}

\author{
Akbas I ${ }^{1}$, Kocak AO ${ }^{2}$, Celik BK ${ }^{2}$, Menekse $\mathrm{TS}^{2}$, Demir $\mathrm{M}^{3}$, Gur STA ${ }^{2}$, Kerget B ${ }^{4}$, Cakir Z ${ }^{2}$ \\ Department of Emergency Medicine, Bingol State Hospital, Bingol, Turkey. akbasilker@gmail.com
}

\section{ABSTRACT}

OBJECTIVES: The aim of this study was to evaluate the diagnostic capacity of integrated pulmonary index $(\mathrm{IPI})$ in predicting the pulmonary embolism (PE) in patients admitted to emergency departments (ED) with dyspnea.

BACKGROUND: The acute dyspnea is one of the most common chief complaints in EDs. PE is a potentially fatal disease and the delay in specific therapy increases the worst outcomes.

METHODS: This study is a prospective methodological study, in which we evaluated the diagnostic performance of the IPI in predicting PE in patients admitted to ED with dyspnea. ROC analysis was used for estimating the accuracy of IPI and OCRS.

RESULTS: Of the 144 patients included in the study, there were $20(13.9 \%)$ PE patients. In the ROC analysis, the best cut-off point for IPI was $\leq 2$. For this cut-off point, the sensitivity and specificity of IPI were $100.0 \%$ and $96.0 \%$, respectively. Besides, the accuracy of IPI was $96.5 \%$ with a +LR of 24.8 and a -LR of 0.0 .

CONCLUSION: IPI was a potential candidate for evaluating the respiratory status, and a limiting tool to prevent unnecessary diagnostic tests and save time in determining the treatment course in dyspneic patients at ED (Tab. 5, Fig. 3, Ref. 34). Text in PDF www.elis.sk

KEY WORDS: dyspnea, pulmonary embolism, integrated pulmonary index, ROC curve, diagnostic test.

\section{Introduction}

Acute dyspnea is one of the most common basic admission complaints to the emergency department (ED) (1). The differential diagnosis of dyspnea includes many disorders at out-patient settings such as cardiovascular disorders, chronic obstructive pulmonary disorders (COPD), lower respiratory tract infections and trauma (1). Although the clinical history of the patient is helpful for healthcare workers in guiding the diagnostic procedure of patients, multiple tests are generally performed to narrow the differential diagnosis of dyspnea $(1,2)$.

Dyspnea is the most common symptom of pulmonary embolism (PE) and PE is included in the differential diagnosis of dyspneic patients admitted to EDs. However, ED staff pay little attention when establishing the diagnostic workup for PE in patients with dyspnea because its prevalence is lower than that of

${ }^{1}$ Department of Emergency Medicine, Bingol State Hospital, Bingol, Turkey, ${ }^{2}$ Department of Emergency Medicine, Faculty of Medicine, Ataturk University, Erzurum, Turkey, ${ }^{3}$ Department of Emergency Medicine, Health Sciences University Bursa Higher Specialization Training and Research Hospital, Bursa, Turkey, and ${ }^{4}$ Department of Pulmonary Diseases, Faculty of Medicine, Ataturk University, Erzurum, Turkey

Address for correspondence: I. Akbas, Bingöl Devlet Hastanesi Acil Servisi, 12100, Bingöl, Turkey.

Phone: +905444222880, Fax: +904422363133 other causes of dyspnea such as pulmonary infections, COPD and cardiovascular illness $(1,2)$. Therefore, when a patient is admitted to ED for an episode of dyspnea, PE is rarely considered as a possible cause. However, PE is a potentially fatal disease, especially in the elderly population, and the delay in specific treatment increases the worst outcomes (3-5).

Many scoring systems are developed for early clinical diagnoses of diseases, especially for diseases that require early diagnosis and prompt intervention due to high morbidity and mortality. These scoring systems foresee patient's clinical worsening and predict in-hospital outcomes (6-8). However, they frequently lack optimal sensitivity and/or specificity to identify patients at high risk $(9,10)$.

Integrated pulmonary index (IPI) is a mathematically-determined factor that incorporates four real-time respiratory parameters: end-tidal $\mathrm{CO}_{2}$, respiratory rate, pulse rate, and $\mathrm{SpO}_{2}$. This index should enable a non-invasive assessment of the respiratory state of the patient $(11,12)$. It was highlighted that all clinicians could use IPI in order to determine whether their patients need additional clinical assessment or intervention (12). Close correlation between IPI and respiratory status of patients who are monitored has been demonstrated $(12,13)$, and it was reported that IPI could be a valuable tool in early determination of respiratory problems at ED (14). IPI score could be used for predicting causes such as pulmonary embolism in patients who were admitted to EDs with severe dyspnea. 
We conducted this study to evaluate the capacity of IPI score to predict PE in patients who were admitted to our ED with dyspnea. The IPI score is a comprehensive formula based only on available four real-time respiratory parameters automatically recorded within a device. We prospectively evaluated this score in a cohort of dyspneic patients managed at the ED to predict PE.

\section{Materials and methods}

\section{Study design and setting}

Our study was a prospective methodological study, in which we studied the diagnostic accuracy of the IPI in predicting pulmonary embolism in patients with dyspnea. We informed all patients about the study and its procedures and collected informed consent on paper from the patients before their inclusion in the study. This research was conducted in accordance with Good Clinical Practice standards, and according to the Standards for the Reporting of Diagnostic Accuracy Studies (STARD) (15). The study was approved by the Ataturk University Faculty of Medicine Ethics Committee and was carried out between 01.09.2019-30.09.2019 at Ataturk University Research Hospital, the major hospital of the Eastern Anatolia Region in Turkey.

\section{Patients}

We evaluated all patients who were admitted to our ED for eligibility. The inclusion criteria were admission to ED with dyspnea (1), and age over 18 years (2). The exclusion criteria were pregnancy (1), having cognitive impairments or psychiatric disorders (2), having any type of cancer (3), presence of bleeding disorder (4), taking medications that increase the risk of bleeding (5), having chronic disease including, diabetes mellitus, hypertension, chronic liver disease, chronic kidney disease, chronic heart disease failure, etc. (6), patients who applied with dyspnea and a computed tomographic pulmonary angiography (CTPA) has not been performed (7).

Age, sex, vital signs (blood pressure, pulse rate, respiratory rate, body temperature, and oxygen saturation) of the patients who agreed to participate in the study were recorded.

\section{Test methods}

IPI scores of the patients were used to predict the severity of short-term development. IPI (Capnostream-20, Medtronic, Israel) is an automated system that was used to assess the patient's respiratory status with an algorithm that simplifies the interpretation of end-tidal $\mathrm{CO}_{2}\left(\mathrm{PetCO}_{2}\right)$, respiration rate, pulse rate, and oxygen saturation $\left(\mathrm{SpO}_{2}\right)$. This assessment is based on a mathematical modelling of these variables to form a single value ranged between 1 and 10 (16). Patients were monitored using Capnostream-20 monitor for IPI scores on admission to the ED, and we recorded the IPI values on the monitor at the 10th minute.

The reference standard of the study was the presence of pulmonary embolism. It was defined as an obstruction of the pulmonary artery or one of its branches detected by CTPA. Toshiba Aquilion One (serial number 6CA1552049/TAEK27896-Japan) was used for CTPA imaging. Computed tomography pulmonary angiograms were interpreted by an experienced radiologist. The "2019 ESC Guidelines for the diagnosis and management of acute pulmonary embolism developed in collaboration with the European Respiratory Society (ERS): The Task Force for the diagnosis and management of acute pulmonary embolism of the European Society of Cardiology (ESC)" was followed for imaging, diagnosis, and treatment of the patients (17). Patients diagnosed with condition other than PE were referred to appropriate differential diagnosis pathways.

\section{Statistical analysis}

Statistical analyses were performed with SPSS version 23 (IBM Corp. in Armonk, NY) and Medcalc version 16 (MedCalc Software bvba, Ostend, Belgium). Descriptive statistics were presented as frequency (n) and percentage (\%) for categorical variables, and median with interquartile range (IQR) for non-normally distributed variables. ROC analysis was used for estimating the accuracy of IPI in predicting pulmonary embolism. The area under ROC curve (AUC) for IPI was calculated, and Delong et al. method was used for calculating the AUC (18). Youden J index was used for estimating the best cut-off values. Sensitivity, specificity, positive likelihood ratio ( $+\mathrm{LR})$, negative likelihood ratio (-LR), and accuracy with $95 \%$ confidence intervals (CIs) were calculated. The value of $p<0.05$ was set as a statistically significance level.

\section{Results}

A total of 8,719 patients were admitted to our ED between 01.09.2019 and 30.09.2019. Of those patients, 8,575 were excluded (8,378 did not meet inclusion criteria, 185 met exclusion criteria, 12 withdrew from the study just after giving information for their consent). Finally, 144 patients were considered eligible for the

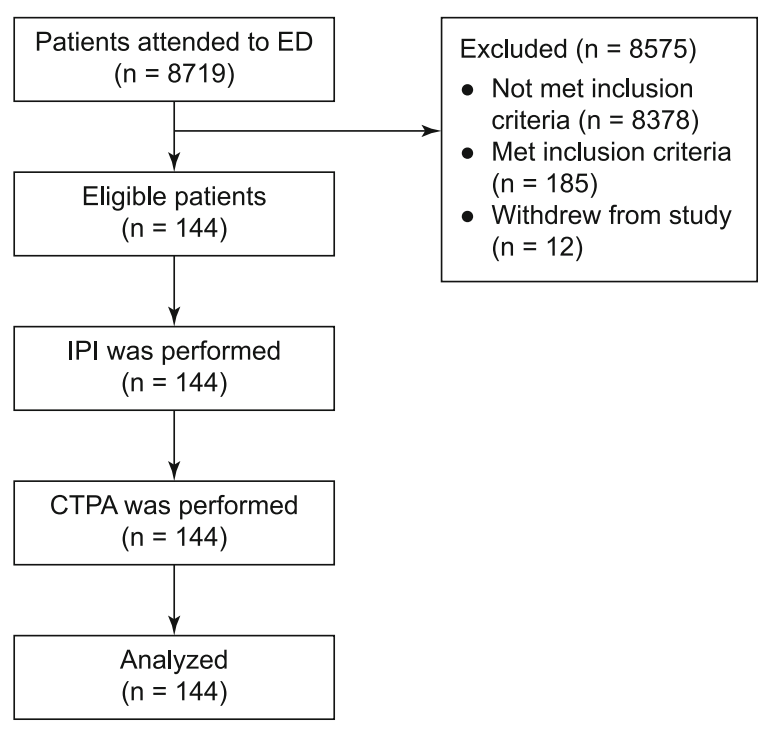

Fig. 1. Flow diagram of the study. IPI - integrated pulmonary index. 
Tab. 1. Demographics and clinical characteristics of the patients.

\begin{tabular}{|c|c|}
\hline \multicolumn{2}{|l|}{ Variables $(n=144)$} \\
\hline Age (years), Median (IQR) & $72.0(67.0-77.0)$ \\
\hline Male, $n(\%)$ & $94(65.3)$ \\
\hline \multicolumn{2}{|l|}{ Arrival examination } \\
\hline Heart rate (BPM), median (IQR) & $88.5(83.0-107.0)$ \\
\hline Respiratory rate (BrPM), median (IQR) & $28.0(23-35)$ \\
\hline Oxygen saturation level (\%), median (IQR) & $88.0(86.0-91.0)$ \\
\hline ETCO $^{2}$ concentration (mmHg), median (IQR) & $31.0(29.0-35.0)$ \\
\hline Systolic blood pressure (mmHg), median (IQR) & $129.0(120.0-142.0)$ \\
\hline Diastolic blood pressure (mmHg), median (IQR) & $74.0(70.0-82.8)$ \\
\hline IPI, median (IQR) & $5.0(3.0-5.0)$ \\
\hline
\end{tabular}

BPM: beats per minute, BrPM: breaths per minute, $\mathrm{ETCO}^{2}$ : end-tidal carbon dioxide, IPI: integrated pulmonary Index

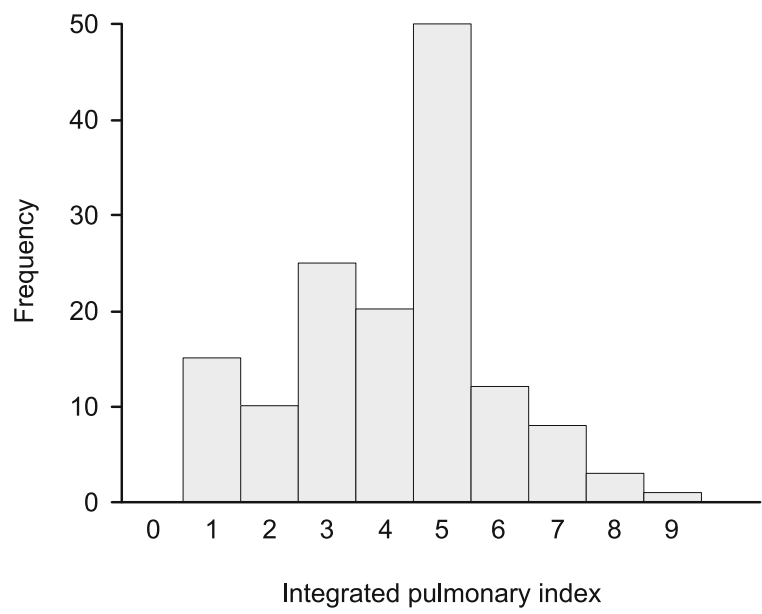

Fig. 2. Distributions of IPI data.

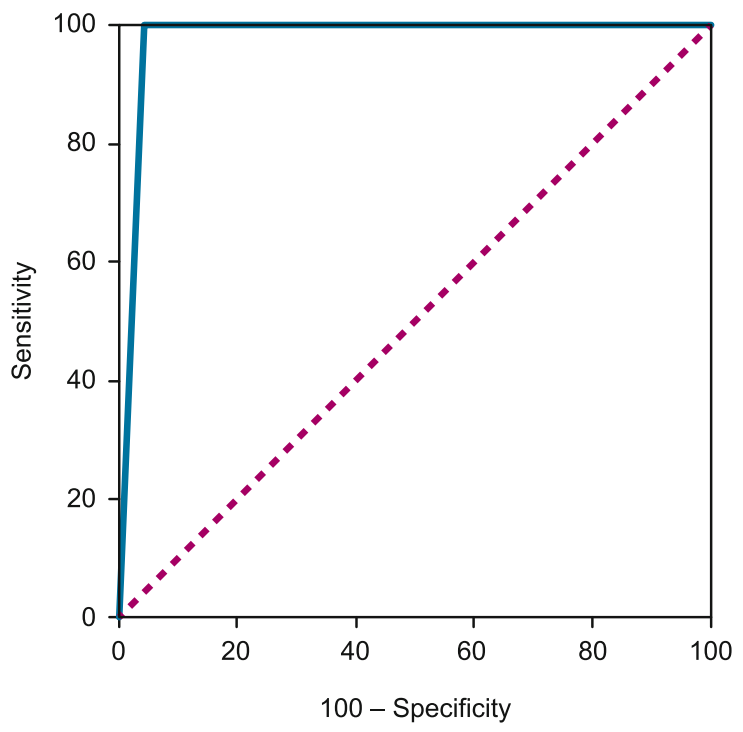

Fig. 3. ROC curve of IPI data.

study. The IPI (integrated pulmonary index) was performed in all of the eligible patients $(n=144)$, and then, all patients underwent CTPA for diagnosing PE (Fig. 1).
Tab. 2. Final diagnosis of the patients.

\begin{tabular}{lr}
\hline Variables $(\mathrm{n}=144)$ & \\
\hline $\begin{array}{l}\text { Pulmonary embolism, } \mathrm{n}(\%) \\
\text { Massive }\end{array}$ & $90(13.9)$ \\
$\quad$ Submassive & $11(7.6)$ \\
\hline COPD, $\mathrm{n}(\%)$ & $44(30.5)$ \\
\hline Pneumonia, $\mathrm{n}(\%)$ & $34(23.6)$ \\
\hline Heart failure, $\mathrm{n}(\%)$ & $25(17.4)$ \\
\hline Others*, $\mathrm{n}(\%)$ & $21(14.6)$ \\
\hline $\begin{array}{l}\text { COPD: chronic obstructive pulmonary disease, } * \text { Other results are acute coronary } \\
\text { syndrome, asthma attack, cancer, anxiety, pleural effusion, pericardial effusion and } \\
\text { pneumothorax }\end{array}$
\end{tabular}

Tab. 3. AUC of the ROC of IPI data.

\begin{tabular}{lcc}
\hline & IPI & $\mathrm{p}$ \\
\hline AUC $(95 \% \mathrm{CI})$ & $0.980(0.941-0.996)$ & $<0.001$ \\
\hline Youden index & 0.960 & \\
\hline Associated cut-off & $\leq 2$ & \\
\hline AUC: area under curve, IPI: integrated pulmonary index &
\end{tabular}

The median age of the patients was 72.0 years, while 94 $(65.3 \%)$ were male. On the baseline examination, the median heart rate was 88.5 beat per minute, the median respiratory rate was 28 breaths per minute, the median oxygen saturation was $88.0 \%$, the median end-tidal carbon dioxide concentration was 31 $\mathrm{mmHg}$, and the median systolic blood pressure was $129.0 \mathrm{mmHg}$ (Tab. 1). The median IPI score was 5.0 (IQR: 3.0-5.0) (Tab. 1 and Fig. 2).

Among the 144 patients who underwent CTPA, 20 (13.9\%) had PE, and of these patients, 9 had massive PE. The other patients were diagnosed with chronic obstructive pulmonary disease, pneumonia, heart failure, or other diseases that may cause dyspnea (coronary syndrome, asthma attack, cancer, anxiety, pleural effusion, pericardial effusion, and pneumothorax) (Tab. 2).

The area under ROC curve (AUC) was 0.980 for IPI. The best cut-off point for IPI, estimated by Youden J index (0.960) was $\leq 2$ (Fig. 3 and Tab. 3). For this cut-off point, the sensitivity and specificity of IPI were 100.0 and 96.0 , respectively. Besides, the accuracy of IPI was 96.5 with a +LR of 24.8 and a - LR of 0.0 (Tab. 4). Also, the comparisons of sensitivities, specificities, +LRs and -LRs of IPI data according to different cut-off values are shown in Appendix Table 5.

\section{Discussion}

$\mathrm{PE}$ is one of the most common causes of death in patients with advanced age and underlying risk factors such as cancer, COPD, and heart failure $(2,19-21)$. The overall annual incidence is estimated to be 100-200 cases per 100,000 individuals (2). The clinical signs of PE are often non-specific; dyspnea is observed in only about $50 \%$ of the patients, and therefore, experts recommend using a prediction algorithm to guide the diagnosis of PE (22-24). Current guidelines recommend the use of validated clinical scores due to the current increase in the number of patients who require sophisticated diagnostic imaging $(2,22)$, while the simplified version of the Wells score is the most frequently used 
Tab. 4. Cross-tabulation of IPI results by the occurrence of PE in the patients with dyspnea.

\begin{tabular}{lccccccc}
\hline Test & \multicolumn{2}{c}{ PE occurrence, $\mathrm{n}(\%)$} & $\begin{array}{c}\text { Sensitivity } \\
(95 \% \mathrm{CI})\end{array}$ & $\begin{array}{c}\text { Specificity } \\
(95 \% \mathrm{CI})\end{array}$ & + LR $(95 \% \mathrm{CI})$ & $-\mathrm{LR}(95 \% \mathrm{CI})$ & Accuracy $(95 \% \mathrm{CI})$ \\
\cline { 2 - 3 } & Not occurred & Occurred & $00.0(83.2-100.0)$ & $96.0(90.8-98.7)$ & $24.8(7.7-80.2)$ & $0.0($ na-na) & $96.5(92.1-98.9)$ \\
\hline $\mathrm{IPI}>2$ & $119(81.0)$ & $0(0.0)$ & 100.0 & & & & \\
\hline $\mathrm{IPI} \leq 2$ & $5(20.0)$ & $20(80.0)$ & & & & \\
\hline
\end{tabular}

IPI: integrated pulmonary index, +LR: positive likelihood ratio, - LR: negative likelihood ratio, na: not available

Tab. 5. Diagnostic performance of IPI according to different cut-off values.

\begin{tabular}{ccccccccc}
\hline Cut-off value & Sensitivity & $95 \%$ CI & Specificity & $95 \%$ CI & + LR & $95 \%$ CI & - LR & $95 \%$ CI \\
\hline$<1$ & 0.0 & $0.0-16.8$ & 100.0 & $97.1-100.0$ & & & 1.0 & $1.0-1.0$ \\
\hline$\leq 1$ & 60.0 & $36.1-80.9$ & 97.6 & $93.1-99.5$ & 24.8 & $7.7-80.2$ & 0.4 & $0.2-0.7$ \\
\hline$\leq 2$ & 100.0 & $83.2-100.0$ & 96.0 & $90.8-98.7$ & 24.8 & $10.5-58.5$ & 0.0 & \\
\hline$\leq 3$ & 100.0 & $83.2-100.0$ & 75.8 & $67.3-83.0$ & 4.1 & $3.0-5.6$ & 0.0 & \\
\hline$\leq 4$ & 100.0 & $83.2-100.0$ & 59.7 & $50.5-68.4$ & 2.5 & $2.0-3.1$ & 0.0 \\
\hline$\leq 5$ & 100.0 & $83.2-100.0$ & 19.4 & $12.8-27.4$ & 1.2 & $1.1-1.4$ & 0.0 & \\
\hline$\leq 6$ & 100.0 & $83.2-100.0$ & 9.7 & $5.1-16.3$ & 1.1 & $1.0-1.2$ & 0.0 & \\
\hline$\leq 7$ & 100.0 & $83.2-100.0$ & 3.2 & $0.9-8.1$ & 1.0 & $1.0-1.1$ & 0.0 & \\
\hline$\leq 8$ & 100.0 & $83.2-100.0$ & 0.8 & $0.02-4.4$ & 1.0 & $1.0-1.0$ & 0.0 & \\
\hline$\leq 9$ & 100.0 & $83.2-100.0$ & 0.0 & $0.0-2.9$ & 1.0 & $1.0-1.0$ & &
\end{tabular}

IPI: integrated pulmonary index, +LR: positive likelihood ratio, -LR: negative likelihood ratio

clinical prediction algorithm for patients with suspected PE (25, 26). It separates patients into two groups according to their score; 4 or fewer points are considered unlikely for PE, and more than 4 are considered likely for PE (26). However, the sensitivity, specificity and accuracy of the basic and other modified versions of Wells score were reported to be 61.5-64.2\%, 61.6-81.0\% and $61.6-69.9 \%$, respectively $(19,22,26,27)$.

To the best of our knowledge, this study is the first to investigate the predictive capacity of IPI scoring to foresee PE in patients admitted to ED with dyspnea. For that purpose, we evaluated the IPI scoring by comparing it with CTPA, which is known as the gold standard diagnostic test for the diagnosis of PE. The IPI score exhibits high predictive performance. The estimated cut-off point for IPI was $\leq 2$. For this cut-off point, the sensitivity and specificity of IPI were 100.0 and 96.0 , respectively. Besides, the accuracy of IPI was 96.5 with a + LR of 24.8 and a - LR of 0.0 . The main aim of our study was to evaluate the capacity of the IPI score of stratifying the risk of PE in the ED setting and we found that IPI had a high predictive accuracy.

The noninvasive measurement of end-tidal $\mathrm{CO}_{2}$ with capnography, which provides information on ventilation, perfusion, and metabolism, demonstrates benefit in patients with seizure, trauma, and respiratory conditions such as pulmonary embolism $(28,29)$. Also, oxygen saturation by pulse oximeter $\left(\mathrm{SpO}_{2}\right)$ has been widely used to detect the early hemodynamic deterioration in patients with several respiratory diseases (30). After all, IPI is a potential candidate to be a more dynamic measurement than the widely used respiratory parameters alone, and therefore determines patient's respiratory status more accurately.

It has been reported that wide heterogeneity was observed in the clinical presentation of PE in the literature. Although the most common presenting symptom is dyspnea, the patients can be admitted to an ED with chest pain and/or syncope $(1,19)$. In addi- tion, wide range of prevalence could be observed in accordance with the selected admission symptom or selected population. The prevalence of PE in our dyspneic cohort of patients undergoing CTPA was $13.9 \%$ and this ratio was compatible with the previously published studies $(19,31)$. Also, our results support the fact that the differences in prevalence may be attributed to the identification of the including symptom at admission.

Clinical probability ratios are basic factors for interpreting the guiding algorithms and making ongoing clinical decisions. Diagnostic scores allow clinicians to safely eliminate a disease without exposing patients to unnecessary diagnostic test methods $(21,27)$. It is known that efficient patient care in EDs significantly decreases the time to diagnosis, treatment, and disposition of patients with PE (21). The previous studies supported that a decrease in time from diagnosis to specific therapy was associated with improved clinical outcomes (4). The presented study showed that the IPI score is helpful to foresee the clinical probability of PE in patients admitted to ED with dyspnea, therefore our results are important for clinicians with a feasible clinical guidance for patients requiring a diagnostic test such as CTPA. By means of the IPI score, we anticipated which of the patients required prompt evaluation by CTPA and prioritized administration of specific therapy.

It is well accepted that the IPI score is quite helpful to closely monitor the patient's respiratory status. Therefore, all clinicians may use IPI score in order to determine which patients need additional clinical assessment and intervention $(12,16)$. IPI is demonstrated as a single indexed value from 1 to 10 , where $8-10$ shows a nearly normal ventilation, whereas a level $\leq 6$ shows that intervention might be required, and a level $\leq 4$ shows that intervention is ultimately necessary $(11,16,32)$. Although, CTPA is the current gold standard diagnostic method, it may not be practical to employ it in all patients who were admitted to EDs with dys- 
pnea and suspected PE $(33,34)$. The CTPA is a time-consuming process and a high proportion of patients receive inappropriate anticoagulant therapy while waiting for this diagnostic approach (20). Early use of IPI score, including basic respiratory parameters, may limit unnecessary diagnostic tests and save time in determining the treatment course. This approach may provide both the best care for the patient and the ED's capability to readily respond to the next patient.

\section{Limitations}

Our study has several limitations. The first limitation is the lack of external validity because it is a single-center study and therefore includes a relatively small sample size. This study represents our experience at a single center in a developing country and may not be representative of other populations. We defined the fact of "not performing a CTPA" as an exclusion criterion because the CTPA is the gold standard for diagnosing PE. On the other hand, including only the patients who underwent CTPA in the study, would have increased the accuracy of IPI for predicting PE because patients were examined for different health problems and underwent different diagnostic and imaging tests according to their pre-diagnoses in ED setting. In other words, the probability of having PE might be higher in the patients who underwent CTPA as compared to the patients who did not. This was also supported by our high pre-test probability of $13.9 \%$. We excluded the patients with cancer and chronic diseases who might have higher risks of PE. The aim here is to restrict these health conditions for eliminating their confounding effects on the association of IPI with PE. Because these health conditions may have possible effects on both IPI measurement and PE occurrence, all these limitations should be kept in the mind when interpreting the results of the study.

\section{Conclusions}

In conclusion, among patients who were admitted to ED due to dyspnea, PE was confirmed in $13.9 \%$ (approximately one in every seven patients). Based on vital parameters available at admission to the ED, the IPI score may have a future role as a triage tool in patients with dyspnea. The IPI score may provide adequate monitoring for patients with dyspnea admitted to the ED.

\section{References}

1. DeVos E, Jacobson L. Approach to Adult Patients with Acute Dyspnea. Emerg Med Clin North Am 2016; 34: 129-149.

2. Pollack CV, Schreiber D, Goldhaber SZ et al. Clinical characteristics, management, and outcomes of patients diagnosed with acute pulmonary embolism in the emergency department: initial report of EMPEROR (Multicenter Emergency Medicine Pulmonary Embolism in the Real World Registry). J Am Coll Cardiol 2011; 57: 700-706.

3. Prandoni P, Lensing AW, Prins MH et al. Prevalence of Pulmonary Embolism among Patients Hospitalized for Syncope. N Engl J Med 2016; 375: 1524-1531.
4. Smith SB, Geske JB, Maguire JM, Zane NA, Carter RE, Morgenthaler TI. Early anticoagulation is associated with reduced mortality for acute pulmonary embolism. Chest 2010; 137: 1382-1390.

5. Weberova D, Weber $\mathbf{P}$, Meluzinova $\mathbf{H}$ et al. Deceased elderly in-patients with pulmonary embolism. Bratisl Lek Listy 2014; 115: 786-790.

6. Viglino D, L'Her E, Maltais F, Maignan M, Lellouche F. Evaluation of a new respiratory monitoring tool "Early Warning ScoreO2" for patients admitted at the emergency department with dyspnea. Resuscitation 2020; 148: 59-65.

7. Smith GB, Prytherch DR, Schmidt PE, Featherstone PI. Review and performance evaluation of aggregate weighted 'track and trigger' systems. Resuscitation 2008; 77: 170-179.

8. Smith GB, Prytherch DR, Schmidt PE, Featherstone PI, Higgins B. A review, and performance evaluation, of single-parameter "track and trigger" systems. Resuscitation 2008; 79: 11-21.

9. Smith ME, Chiovaro JC, O’Neil M et al. Early warning system scores for clinical deterioration in hospitalized patients: a systematic review. Ann Am Thorac Soc 2014; 11: 1454-1465.

10. Maharaj R, Raffaele I, Wendon J. Rapid response systems: a systematic review and meta-analysis. Crit Care 2015; 19: 254.

11. Riphaus A, Wehrmann T, Kronshage $T$ et al. Clinical value of the Integrated Pulmonary $\operatorname{Index}((\mathrm{R}))$ during sedation for interventional upper GI-endoscopy: A randomized, prospective tri-center study. Dig Liver Dis 2017; 49: 45-49.

12. Mermer D, Turan G, Koksal C, Tas BA, Karadogan F, Akgun N. Correlation of integrated pulmonary index with clinical observation in unilateral and bilateral spinal anaesthesia in geriatric patients. Internat $\mathrm{J}$ Res Med Sci 2016; 4 (10).

13. Kumar V, Taft A, Herrington R, Whiddon S, Castresana M. Integrated pulmonary index for successful weanıng obese patients after cardiac bypass surgery 2013; 41: A90.

14. Kuzkov VV, Gaidukov KM, Fot EV, Neverova MS, Smetkin AA, Kirov MY. Integrated pulmonary index reflects respiratory function after elective coronary artery bypass grafting: 5AP4-5 2011; $28: 78$.

15. Cohen JF, Korevaar DA, Altman DG et al. STARD 2015 guidelines for reporting diagnostic accuracy studies: explanation and elaboration. BMJ Open 2016; 6: e012799.

16. Ronen M, Weissbrod R, Overdyk FJ, Ajizian S. Smart respiratory monitoring: clinical development and validation of the IPI (Integrated Pulmonary Index) algorithm. J Clin Monit Comput 2017; 31: 435-442.

17. Konstantinides SV, Meyer G, Becattini C et al. ESC Guidelines for the diagnosis and management of acute pulmonary embolism developed in collaboration with the European Respiratory Society (ERS): The Task Force for the diagnosis and management of acute pulmonary embolism of the European Society of Cardiology (ESC). Eur Respir J 2019; 54.

18. DeLong ER, DeLong DM, Clarke-Pearson DL. Comparing the areas under two or more correlated receiver operating characteristic curves: a nonparametric approach. Biometrics. 1988; 44: 837-845.

19. Spampinato MD, Bucci S, Migliano MT et al. Assessing Pretest Clinical Risk of Pulmonary Thromboembolism in the Emergency Department: Proposal of a Simple Modification to the Wells' Score. J Emerg Med 2020.

20. Konstantinides SV, Torbicki A, Agnelli G et al. ESC guidelines on the diagnosis and management of acute pulmonary embolism. Eur Heart J 2014; 35: 3033-3069. 
$65-70$

21. Wright C, Elbadawi A, Chen YL et al. The impact of a pulmonary embolism response team on the efficiency of patient care in the emergency department. J Thromb Thrombolysis 2019; 48: 331-335.

22. Posadas-Martinez ML, Vazquez FJ, Giunta DH, Waisman GD, de Quiros FG, Gandara E. Performance of the Wells score in patients with suspected pulmonary embolism during hospitalization: a delayed-type cross sectional study in a community hospital. Thromb Res 2014; 133: 177-181.

23. Le Gal G, Righini M, Roy PM et al. Prediction of pulmonary embolism in the emergency department: the revised Geneva score. Ann Intern Med 2006; 144: 165-171.

24. Roy PM, Colombet I, Durieux P, Chatellier G, Sors H, Meyer G. Systematic review and meta-analysis of strategies for the diagnosis of suspected pulmonary embolism. Bmj 2005; 331: 259.

25. Ceriani E, Combescure C, Le Gal G et al. Clinical prediction rules for pulmonary embolism: a systematic review and meta-analysis. J Thromb Haemost 2010; 8: 957-970.

26. Wells PS, Anderson DR, Rodger $M$ et al. Derivation of a simple clinical model to categorize patients probability of pulmonary embolism: increasing the models utility with the SimpliRED D-dimer. Thromb Haemost 2000; 83: 416-420.

27. Wells PS, Ginsberg JS, Anderson DR et al. Use of a clinical model for safe management of patients with suspected pulmonary embolism. Ann Intern Med. 1998; 129: 997-1005.
28. Long B, Koyfman A, Vivirito MA. Capnography in the Emergency Department: A Review of Uses, Waveforms, and Limitations. J Emerg Med 2017; 53: 829-842.

29. Nassar BS, Schmidt GA. Capnography During Critical Illness. Chest 2016; 149: 576-585.

30. Pieraccini M, Guerrini S, Laiolo E et al. Acute Massive and Submassive Pulmonary Embolism: Preliminary Validation of Aspiration Mechanical Thrombectomy in Patients with Contraindications to Thrombolysis. Cardiovasc Intervent Radiol 2018; 41: 1840-1848.

31. Miniati M, Prediletto R, Formichi B et al. Accuracy of clinical assessment in the diagnosis of pulmonary embolism. Am J Respir Crit Care Med. 1999; 159: 864-871.

32. Kaur R, Vines DL, Liu L, Balk RA. Role of Integrated Pulmonary Index in Identifying Extubation Failure. Respir Care 2017; 62: 1550-1556.

33. Raja AS, Ip IK, Prevedello LM et al. Effect of computerized clinical decision support on the use and yield of CT pulmonary angiography in the emergency department. Radiology 2012; 262: 468-474.

34. Vinson DR, Mark DG, Chettipally UK et al. Increasing Safe Outpatient Management of Emergency Department Patients With Pulmonary Embolism: A Controlled Pragmatic Trial. Ann Intern Med 2018; 169: 855-865.

Received June 24, 2020. Accepted July 14, 2020. 